\title{
Opportunity cooperative embedded platform and control mechanism based on selectivity of time and frequency domain
}

\author{
Yong $\operatorname{Jin}^{1 *}$, Huan Dai ${ }^{1}$, Canghai Sui ${ }^{1}$, Anqi Liu ${ }^{1}$ and Ping $\mathrm{Xu}^{2}$
}

\begin{abstract}
The opportunistic cooperative platform and adaptive cooperative control scheme were proposed based on the redundancy degree and priority of cooperative data packet, as well as selective characteristics of time domain and frequency domain. First, opportunistic cooperative platform was defined according to the cooperative data stream scale, mapping algorithm, and priority and redundancy of cooperative data packets. Second, under the single or multiple relay network environment and mobility of sensors, the selective characteristics of time and frequency domain were studied. The active domain and negative domain were proposed for adjusting and improving the opportunistic cooperative platform. Third, the opportunistic cooperative control mechanism based on selectivity of time domain and frequency domain (OCC-TFD) was proposed for improving the performance of wireless sensor networks. Finally, the mathematical results demonstrated that the proposed platform and scheme can provide reliable, robust, and efficient data communication services of wireless sensor networks.
\end{abstract}

Keywords: Opportunistic cooperative embedded platform, Opportunistic cooperative embedded control, Selectivity of time domain, Selectivity of frequency domain, Wireless sensor networks, Services guarantee

\section{Introduction}

The opportunistic network is composed of the nodes with self-organized data transmission without maintaining the transmission path between the sender and destination nodes, which give full consideration to the dynamic characteristics [1] of the network topology. However, in the cooperative communications [2], the sensors cannot provide the reliable and real-time performance of remote, long-time, and large amount of data because of the interference restrictions such as power source, hardware equipment noise [3], and unknown environment factor [4]. Specially, the data stream has the following features. There are the different requirements of data stream structure for data storage [5], data processing, and transmission. The user requirements have the diversity, and the network state is the dynamic change. So, the previous static opportunistic network algorithm [6], data stream computing method, and fixed

\footnotetext{
* Correspondence: jinyong@cslg.cn

${ }^{1}$ School of Computer Science and Engineering, Changshu Institute of

Technology, Changshu 215500, China

Full list of author information is available at the end of the article
}

cooperation transmission method of cooperative communication algorithm have been unable to satisfy the diversity of data stream application over wireless sensor networks. Consequently, how to combine the opportunity network technology and cooperative communication technology has been the widespread concern in academia and industry, which should consider the network topology and data stream characteristics and establishment of cooperative control mechanism.

On the one hand, based on the independent and nonidentically distributed Nakagami-m fading channels, Yuan et al. [7] researched the dual-hop wireless cooperative network with opportunistic amplify-and-forward relaying algorithm. Shi et al. [8] proposed the multi-user cooperative communication protocol for selecting the relay node and improving the system throughput, based on the opportunistic communication and network coding. Based on the opportunistic analysis of a broader class of networks, the new opportunistic methods for several network geometries was proposed [9]. The low overhead multi-relay selection protocol was proposed to support multi-stream cooperative communications in [10]. Then, the diversity-multiplexing 
tradeoff and the throughput-reliability tradeoff were discussed. According to the issue of opportunistic data transfer with mobile ad hoc networks, the cooperative opportunistic routing in mobile ad hoc networks was proposed by Wang et al. [11] which is a pure network layer scheme.

On the other hand, Wu et al. [12] proposed the use of smartphones to collect data from sensor nodes opportunistically, by accessing data from a wireless sensor network and over the generation of data through participatory sensing. A single-hop wireless sensor network was researched over the sensor nodes and the controller node which have multiple antennas [13]. The information theoretical criterion for opportunistic sensing was proposed by Liang et al. [14], which proved that wireless heterogeneous sensor network with correlated modalities needs less number of code words than that with independent modalities. The homeland defense use case for opportunistic networks was simulated [15], which involves detecting a suspicious watercraft. The drawback with the current short-range neighbor discovery technology was discussed in opportunistic networks [16]. The dynamic cooperative routing was proposed [17], which could modify equip with an adaptive decision-making mechanism.

The rest of this paper is organized as follows. Section 2 gives the definition of opportunistic cooperation platform. Section 3 gives the selective characteristics of time and frequency domain. Section 4 gives the cooperative control algorithm. Section 5 studied and evaluated the performance of the proposed platform and scheme to prove the validity and feasibility of the proposed algorithm. Finally, the conclusion is made in Section 6.

\section{Opportunity cooperation platform}

Let $M$ denote the relay sensors set of wireless sensor networks. $T$ is the qualitative parameter set of cooperative data stream $X$. When $X$ belongs to $M$ and $T$ is random mapping, the determining degree $f(x)$ belongs to the interval $[0,1]$, which is a random number with a stable trend. Hence, opportunity cooperation platform $M(X)$ is defined as the distribution of $X$ on $M$.

The $n$ data packets of $X$ are stored in the $M(X)$, which is defined as $P=P_{1}, P_{2} \ldots P_{n}$. Each data packet may belong to one, two, or more opportunistic cooperative nodes. The candidate data packet $P_{i}$ could be calculated by Eq. (1) based on user demand $U_{r}$ and $T$.

$$
P_{i}=\left\{\begin{array}{c}
T_{p}\left(P_{i}\right) \\
h\left(P_{i}, T\right) \\
\phi\left(T_{p}, f\left(P_{i}\right) h\left(P_{i}, T\right), U_{r}\right)
\end{array} \quad 0<i \leq n\right.
$$

Here, let $h\left(F_{i}, T\right)$ denote the analysis function of data packets with the qualitative parameters. Let $\phi\left(T_{p}\right.$, $\left.f\left(F_{i}\right) h\left(F_{i}, T\right), U_{r}\right)$ denote the combination of the above analysis results and user demand. Specially, combination of the determined degree $f\left(F_{\mathrm{i}}\right)$ of data packets and qualitative analysis $h\left(F_{\mathrm{i}}, T\right)$ could make the user mapping opportunistic data stream accurate.

Specially, let $T_{p}\left(F_{i}\right)$ denote the priority determined by cooperative data packets, which could be analyzed according to the calculation results of Eq. (2).

$$
T_{p}\left(P_{i}\right)=\left\{\begin{array}{l}
1, \frac{1}{L_{\mathrm{M}}} \sum_{k=1}^{L_{\mathrm{M}}} \alpha M_{k}\left(P_{i}\right)<f\left(P_{i}\right) \\
2, \frac{1}{L_{\mathrm{M}}} \sum_{k=1}^{L_{\mathrm{M}}} \alpha M_{k}\left(P_{i}\right)=f\left(P_{i}\right) \\
3, \frac{1}{L_{\mathrm{M}}} \sum_{k=1}^{L_{\mathrm{M}}} \alpha M_{k}\left(P_{i}\right)>f\left(P_{i}\right)
\end{array}\right.
$$

Here, $L_{\mathrm{M}}$ denotes the length of $M$ set. Let $\alpha$ denotes the redundancy coefficient of cooperative data packets, which could be obtained by Eq. (3).

$$
\alpha=\left\{\begin{array}{l}
1, P_{i} \in M\left(P_{i}\right) \\
0, P_{i} \notin M\left(P_{i}\right)
\end{array}\right.
$$

The priority of cooperative data stream $X$ could be calculated by Eq. (4).

$$
S(X)=\left\{\begin{array}{l}
1, \frac{1}{L_{\mathrm{X}}} \sum_{k=1}^{L_{\mathrm{X}}} T_{p}\left(P_{k}\right)<f(X) \\
2, \frac{1}{L_{\mathrm{X}}} \sum_{k=1}^{L_{\mathrm{X}}} T_{p}\left(P_{k}\right)=f(X) \\
3, \frac{1}{L_{\mathrm{X}}} \sum_{k=1}^{L_{\mathrm{X}}} T_{p}\left(P_{k}\right)>f(X)
\end{array}\right.
$$

Here, $L_{\mathrm{X}}$ is the data packet number of $\mathrm{X}$.

When $S(X)$ is 1 , the cooperative data packets of cooperative data stream are distributed uniformly in the relay nodes of $M$, which would be defined by the primary cooperative data streaming $S_{\mathrm{P}}$. When $S(X)$ is 2, the cooperative data packets of cooperative data stream are centrally located in some of the relay nodes of $M$, which would be defined by the secondary cooperative data streaming $S_{\mathrm{S}}$. When $S(X)$ is 3 , the cooperative data packets of cooperative data stream are highly concentrated in some of the relay nodes of $M$, which would be defined by the advanced cooperative data streaming $S_{\mathrm{A}}$. They are the following relationships between determining degree and three-level cooperative data stream.

$$
g(x)=T_{p}\left(P_{i}\right) \exp \left(-\frac{L\left(S_{1}\right)}{L\left(S_{L}\right)+L\left(S_{H}\right)}\right)
$$

Here, let $L\left(S_{\mathrm{P}}\right), L\left(S_{\mathrm{S}}\right)$, and $L\left(S_{\mathrm{A}}\right)$ represent the length of $S_{\mathrm{P}} S_{\mathrm{S}}$, and $S_{\mathrm{A}}$, respectively, the unit of which are number of packets. They have the following characteristics: 
1) Passive tag means that the wireless sensor networks upload the cooperative data stream and adaptive mark some opportunistic cooperative nodes with the establishment of cooperative transport service session.

2) Active on-demand service is to provide the cooperative data transmission services of a sender and a receiver based on user needs of active wireless sensor networks.

3) Opportunity cooperative communication with data streaming.

4) Reliability of cooperative communication means that with high reliability, real-time and robust data streaming service would be provided for users.

\section{Performance study of cooperation communication based on selectivity of time domain and frequency domain}

In the cooperative communication of wireless sensor networks, the receiver could obtain the direct path signal and cooperative path signal with different relay nodes. We researched and studied the performance of cooperation communication with single relay, multiple relay, frequency domain, and time domain.

The single-relay communication is given by Fig. 1 with the static state of the receiver. The delay from the sender to receiver $\mathrm{D}$ with the direct path signal is $r_{0} / c$. The delay with cooperative path signal is $\left(r_{1}+r_{2}\right) / c$. Here, $r_{2}$ $=\sqrt[2]{r_{0}^{2}+r_{1}^{2}-2 r_{0} r_{1} \cos \theta}$. Hence, the synthetic signal of $\mathrm{D}$ node is shown as Eq. (6). Here, node $S$ is the sender and node $\mathrm{R}$ is the relay node.

$$
\begin{aligned}
E(t)= & \frac{1}{r_{0}} \cos 2 \pi f\left(t-\frac{r_{0}}{c}\right) \\
& +\frac{1}{r_{1}+r_{2}} \cos 2 \pi f\left(t-\frac{r_{1}+r_{2}}{c}\right)
\end{aligned}
$$

The direct radial signal, cooperative signal, and received synthetic signal with time and frequency are illustrated as Fig. 2. Here, $f$ is the emission frequency of the sender or relay nodes. According to the results of Fig. 2, we can get the following conclusions.

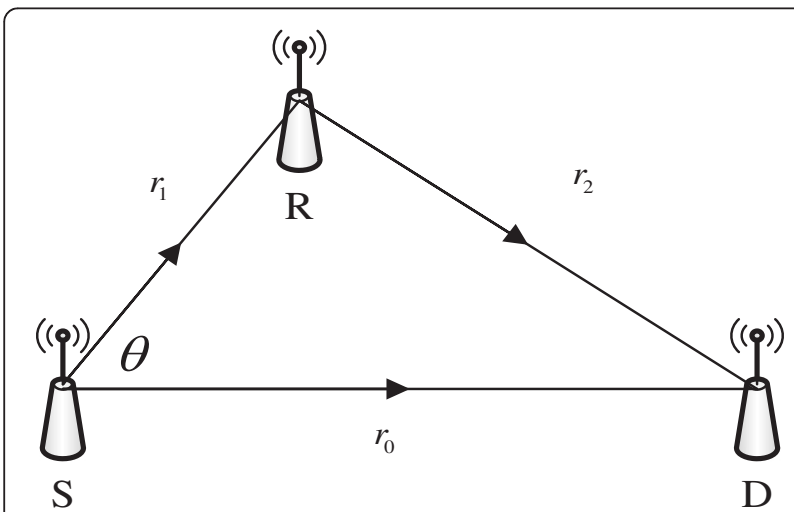

Fig. 1 Communication topology of single relay
1) When $f$ is $0.5 \mathrm{~Hz}$ and time is $0.5 \mathrm{~s}$, the signal phase changed. Here, the synthetic signal is superior to the direct path signal with lower cooperative path signal and less phase change.

2) When $f$ is $1 \mathrm{~Hz}$ and time is $0.5 \mathrm{~s}$, the receiving signal reached the trough and the synthesized signal is always greater than the direct path signal and the synthetic signal when $f$ is 0.5 .

3) When $f$ is $2 \mathrm{~Hz}$ and time is $0.5 \mathrm{~s}$, the signal reached the peak. Here, the cooperative path signal and the changing frequency are larger than one with $f$ is $1 \mathrm{~Hz}$.

4) When $f$ is $10 \mathrm{~Hz}$, the synthetic signal is larger than the above three schemes. The three signals are always keeping a stable trend.

In summary, the better reception signal could be got with the lower direct signal and relay signals. We found that the phase variation of the intensity and the existing frequency boundary value could set up the frequency domain cooperation selective range for choosing reasonable relay node optimization synthesis signal at the receiving end. So, we give the following definition of signal relay topology.

1) Negative cooperative frequency domain when $f$ belongs to the interval $[0.5,10] \mathrm{Hz}$. Here, the synthetic signal could be enhanced by increasing the frequency. In addition, decreasing frequency could smooth the phase change of the cooperative path signal.

2) Active cooperative frequency domain when $f$ is larger than $10 \mathrm{~Hz}$, which not only can enhance the synthesized signal significantly but also can smooth the phase change of the cooperative signal completely.

Specially, if the synthesized signal intensity cannot satisfy the user needs or provide reliable transmission for data communications, then it is transferred to the multirelay scheme.

The multiple relays communication is given by Fig. 3 with the static state of the receiver.

The delay from sender to receiver $\mathrm{D}$ with the direct path signal is $r_{0} / c$. The delay with signal of cooperative path 1 is $\left(r_{1}+r_{2}\right) / c$. Here, $r_{2}=\sqrt[2]{r_{0}^{2}+r_{1}^{2}-2 r_{0} r_{1} \cos \theta_{1}}$. The delay with signal of cooperative path 2 is $\left(r_{3}+r_{4}\right) / c$. Here, $r_{4}=\sqrt[2]{r_{0}^{2}+r_{3}^{2}-2 r_{0} r_{3} \cos \theta_{2}}$. Hence, the synthetic signal of D node is shown as Eq. (7).

$$
\begin{aligned}
E(t)= & \frac{1}{r_{0}} \cos 2 \pi f\left(t-\frac{r_{0}}{c}\right)+ \\
& \frac{1}{r_{1}+r_{2}} \cos 2 \pi f\left(t-\frac{r_{1}+r_{2}}{c}\right)+ \\
& \frac{1}{r_{3}+r_{4}} \cos 2 \pi f\left(t-\frac{r_{3}+r_{4}}{c}\right)
\end{aligned}
$$

The direct radial signal, cooperative signal, and received synthetic signal with time and frequency are 


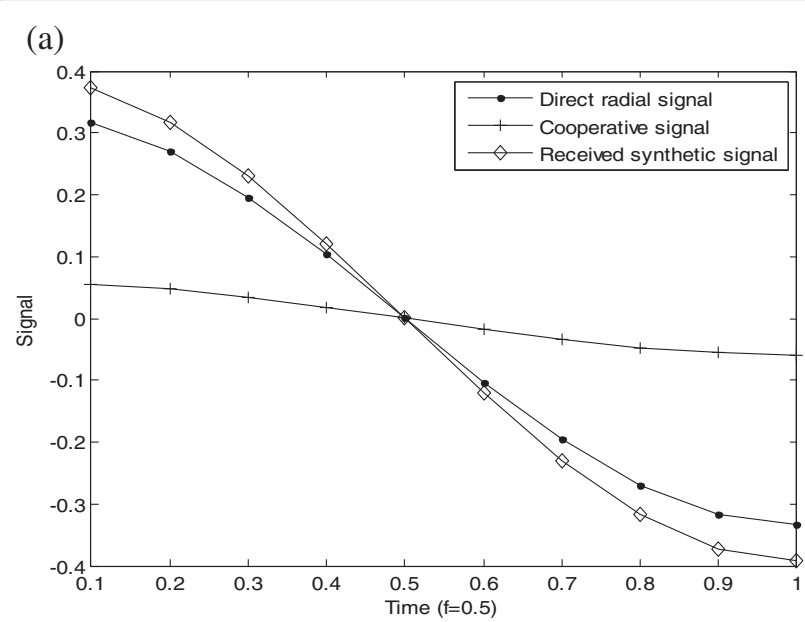

(b)

(c)
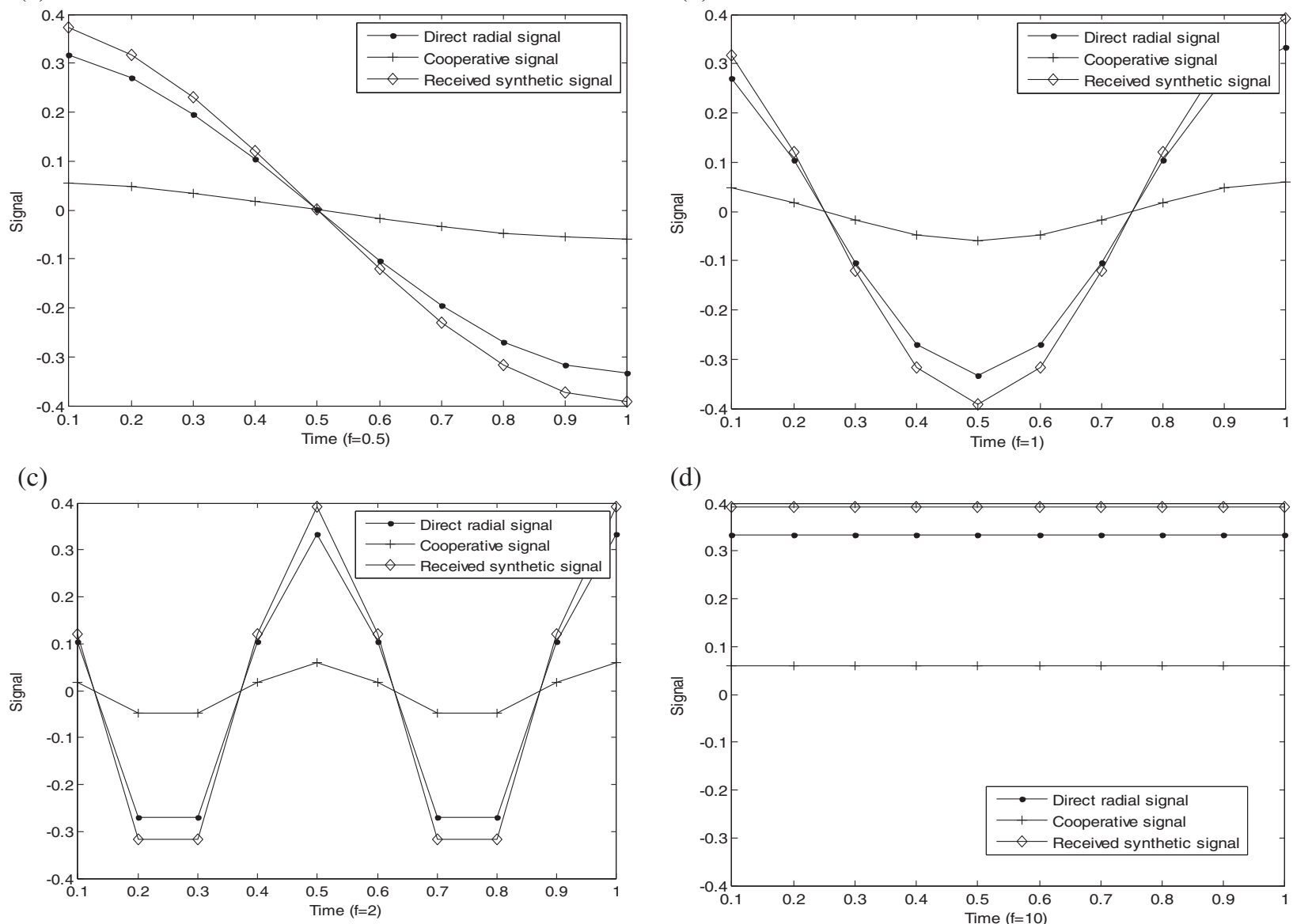

(d)

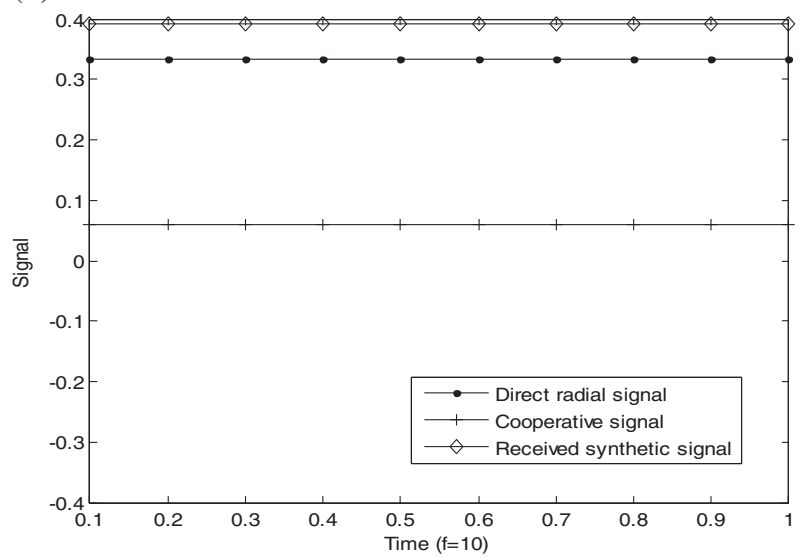

Fig. 2 Direct radial signal, cooperative signal, and received synthetic signal with time and frequency. a $f=0.5 \mathrm{~Hz}$. b $f=1 \mathrm{~Hz}$. $\mathbf{c} f=2 \mathrm{~Hz}$. $\mathbf{d} f=10 \mathrm{~Hz}$

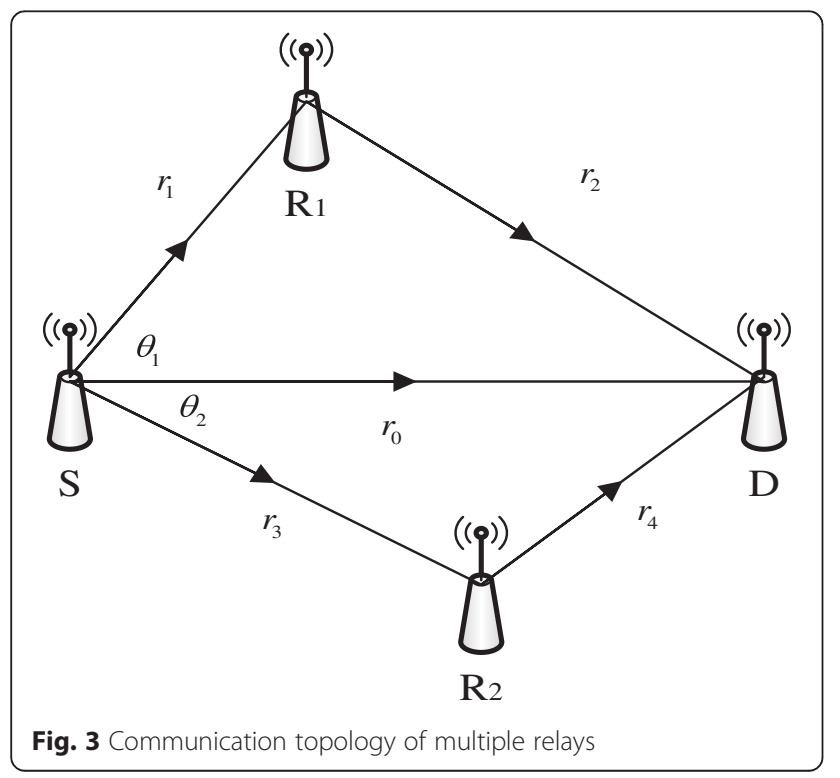

illustrated as Fig. 4. Here, $f$ is the emission frequency of sender or relay nodes. According to the results of Fig. 4, we can get the following conclusions.

1) When $f$ is $0.5 \mathrm{~Hz}$ and time is $0.5 \mathrm{~s}$, the signal phase changed. Here, the cooperative path signal has to be enhanced for obtaining the better synthetic signal.

2) When $f$ is $1 \mathrm{~Hz}$ and time is $0.5 \mathrm{~s}$, the signal reached wave trough.

3) When $f$ is $2 \mathrm{~Hz}$, the phase change is two times one with $f$ is $1 \mathrm{~Hz}$. Specially, the synthetic signal is smaller than the above schemes.

4) When $f$ is $10 \mathrm{~Hz}$, the synthetic signal is larger than the above three schemes, which is superior to the signal with single-relay scheme.

In summary, in the multi-relay scheme, increasing frequency would increase the frequency of phase change, which is caused by multipath propagation. The synthetic signal strength decreases. But the signal tends to smooth, and the intensity is significantly higher than that of single 

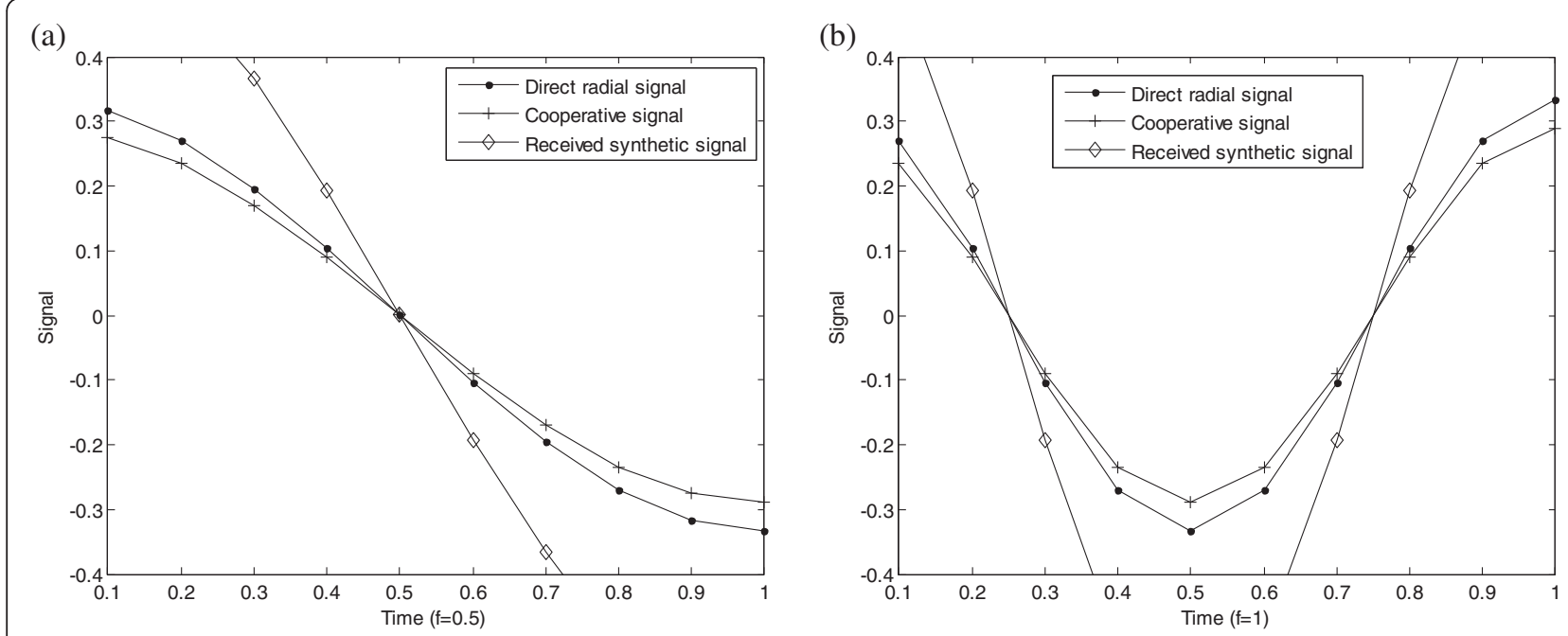

(c)

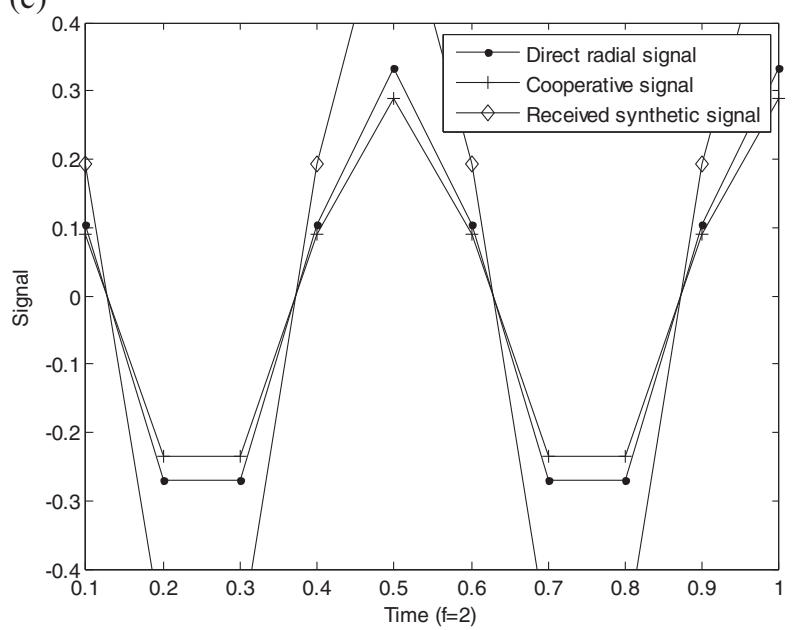

(d)

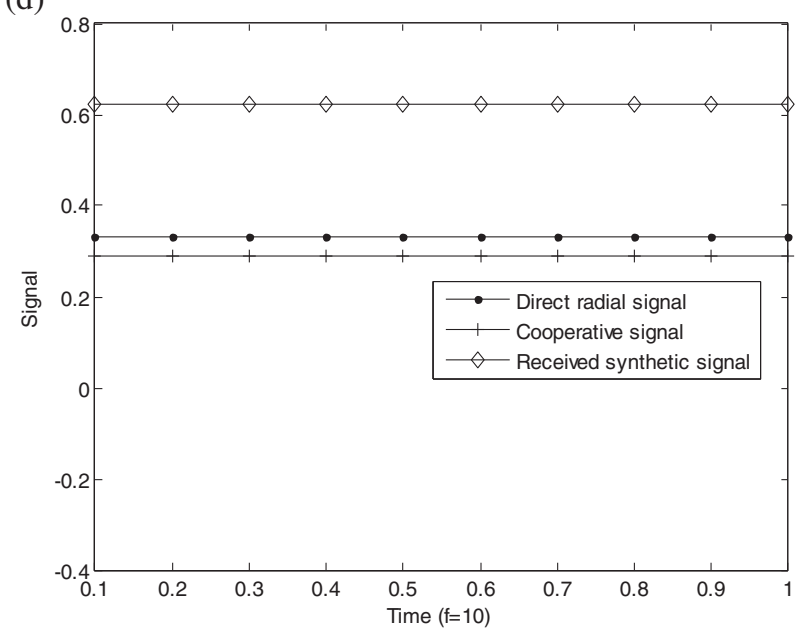

Fig. 4 a $f=0.5 \mathrm{~Hz}$. $\mathbf{b} f=1 \mathrm{~Hz} . \mathbf{c} f=2 \mathrm{~Hz} . \mathbf{d} f=10 \mathrm{~Hz}$

relay after the frequency increases ten times. So, we give the following definition of multiple-relay topology.

1) Negative cooperative frequency domain when $f$ belongs to the interval $[0.5,10] \mathrm{Hz}$. Here, the synthetic signal could be larger than the direct path signal by decreasing the frequency. In addition, decreasing frequency could smooth the phase change of cooperative path signal.

2) Active cooperative frequency domain when $f$ is larger than $10 \mathrm{~Hz}$, which is similar to the single-relay scheme.

The influence of the angle of the arc on the performance is shown in Fig. 5. When the arc is larger, the signal is larger, and the synthetic signal is weakened at any angle. But when $f$ is $10 \mathrm{~Hz}$, the arc angle of the cooperative path does not have any effect.

In order to strengthen the intensity of the synthesized signal, the relay nodes with the large angle arc should be chosen to cooperate in the frequency domain. The positive frequency domain does not affect.

The analytical results of the direct radial signal, cooperative signal, and received synthetic signal with single-relay network topology are illustrated as Fig. 6. According to the results of Fig. 6, we can get the following conclusions.

1) When $f$ is $0.5 \mathrm{~Hz}$ and time belongs to interval [0, 3], the synthetic signal is larger than direct path signal and cooperative path signal. So, the relay nodes should be active for cooperation transmission. However, in interval $[3,5]$, the synthetic signal is less than the cooperative path signal. Here, the transmission wasted the cooperation gain. So, the relay nodes should be negative for saving relay resources and improving utilization ratio. Similarly, we can obtain the active or negative time interval when $f$ is $1,1.5,2,2.5$, and $100 \mathrm{~Hz}$. 

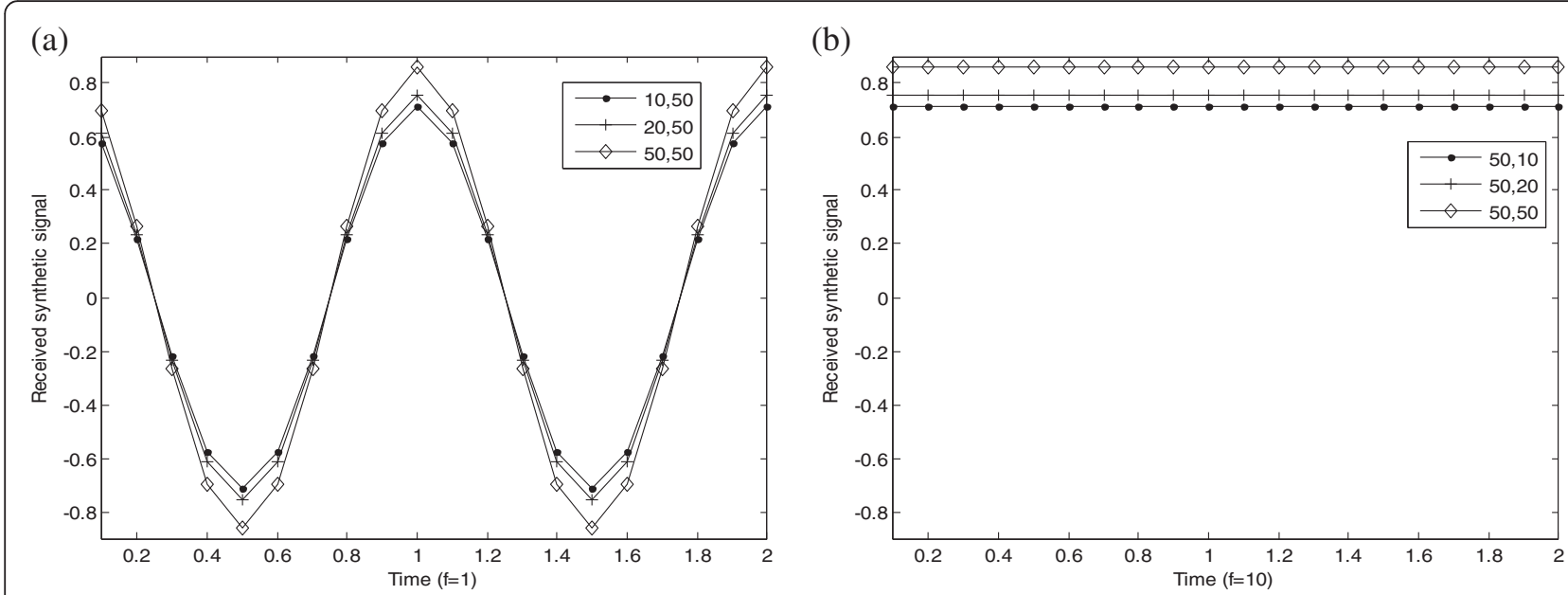

Fig. 5 a $f=1 \mathrm{~Hz}$. $\mathbf{b} f=10 \mathrm{~Hz}$

2) When $f$ is $1 \mathrm{~Hz}$, interval $[0,1.5] \mathrm{s}$ is the active cooperative domain. Then, interval $[1.5,3.5] \mathrm{s}$ is the negative cooperative domain. The interval $[3.5,5] \mathrm{s}$ is the active cooperative domain.

3) When $f$ is $1.5 \mathrm{~Hz}$, interval $[0,1] \mathrm{s}$ is the active cooperative domain. Then, interval $[1.5,2] \mathrm{s}$ is the negative cooperative domain. The interval $[2.5,3] \mathrm{s}$ is the active cooperative domain. The interval [3.5, $5] \mathrm{s}$ is negative cooperative domain.

4) When $f$ is $2 \mathrm{~Hz}$, interval $[0,1] \mathrm{s}$ is the active cooperative domain. Then, interval $[1,1.5] \mathrm{s}$ is the negative cooperative domain. The interval $[1.5,3] \mathrm{s}$ is the active cooperative domain. The interval [3.5, $5] \mathrm{s}$ is negative cooperative domain.

5) When $f$ is $2.5 \mathrm{~Hz}$, interval $[0,1] \mathrm{s}$ is the active cooperative domain. Then, interval $[1,1.5] \mathrm{s}$ is the negative cooperative domain. The interval $[1.5,2.5]$ $\mathrm{s}$ is the active cooperative domain. The interval [2.5, 5] $\mathrm{s}$ is negative cooperative domain.

6) When $f$ is $100 \mathrm{~Hz}$, the full time domain is the active cooperative domain.

According to the different frequency values, the active cooperative time domain and the negative cooperative time domain are defined, for obtaining the effective gain and the maximum resource utilization.

The analytical results with multiple relay network topology are illustrated as Fig. 7. According to the results of Fig. 7 , we can get the following conclusions.

1) When $f$ is $1.5 \mathrm{~Hz}$, interval $[0,1] \mathrm{s}$ is the active cooperative domain. Then, interval $[1,2] \mathrm{s}$ is the negative cooperative domain.

2) When $f$ is $2.5 \mathrm{~Hz}$, interval $[0,0.3] \mathrm{s}$ is the negative cooperative domain. Then, interval $[0.3,0.7] \mathrm{s}$ is the active cooperative domain. The interval $[0.7,1.3] \mathrm{s}$ is the negative cooperative domain. The interval [1.3, 2] $\mathrm{s}$ is active cooperative domain.

3) When $f$ is $10 \mathrm{~Hz}$, the full time domain is the active cooperative domain. In addition, there are phase changes.

4) When $f$ is $100 \mathrm{~Hz}$, the full time domain is the active cooperative domain and the signal phase keeps stable.

In summary, the multiple relay and single relay are the same, but the specific time interval is different. The specific time domain must be determined by real-time analysis.

\section{Cooperative control mechanism}

Based on the definition of opportunistic cooperative nodes and analytical results of characteristics of frequency and time with the received synthetic signal, opportunistic cooperative network platform and control scheme can be built as the following algorithms, which are illustrated as Figs. 8 and 9.

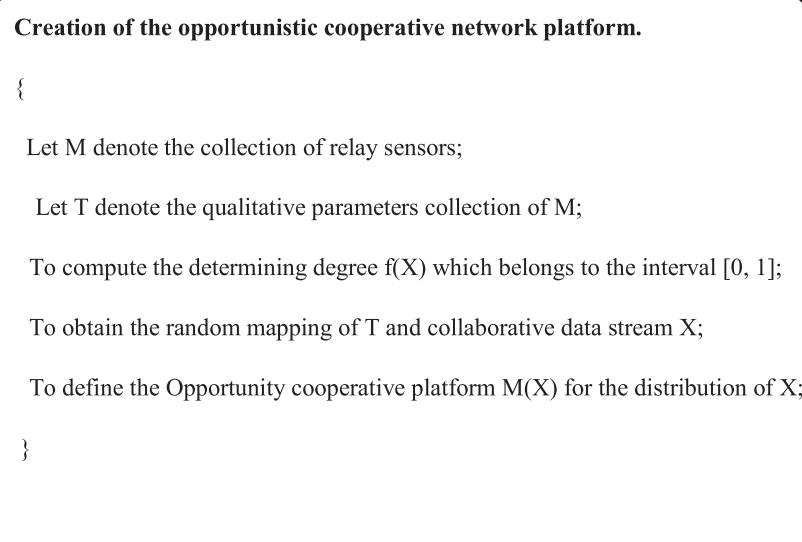




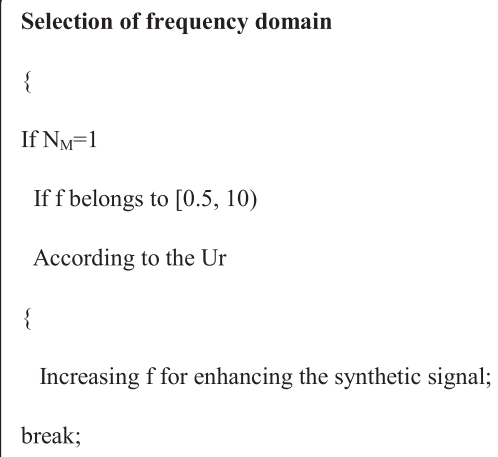

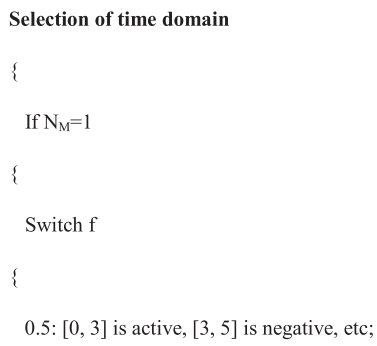

In the process of opportunistic cooperation communication, opportunistic cooperation platform status could be defined as $\left\{N_{\mathrm{M}}, \mathrm{SNR}, \mathrm{TD}, \mathrm{FD}, P_{\mathrm{b}}\right\} . N_{\mathrm{M}}$ is the scale of opportunistic cooperation cloud. Let TD denote time selection. Let FD denote frequency selection. 

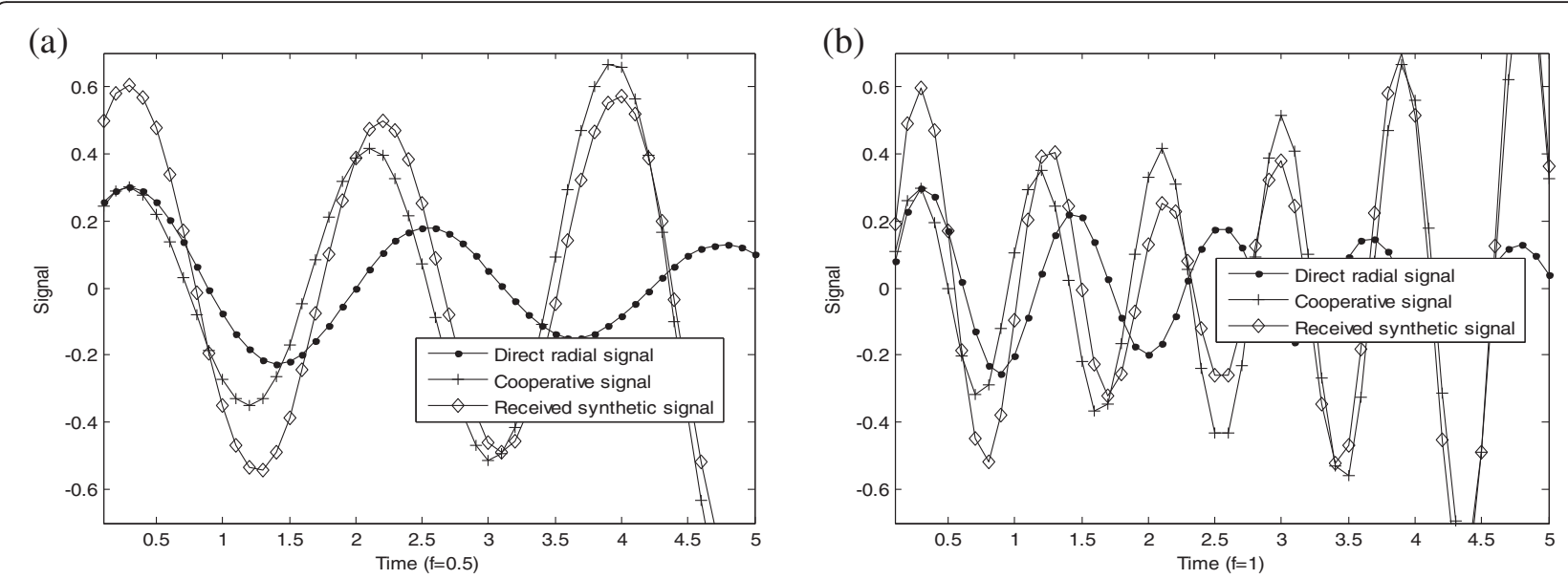

(c)

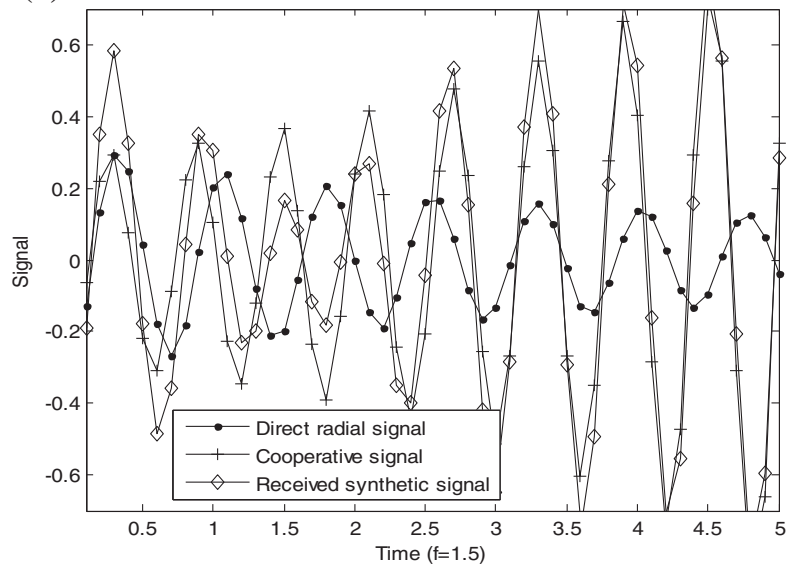

(d)

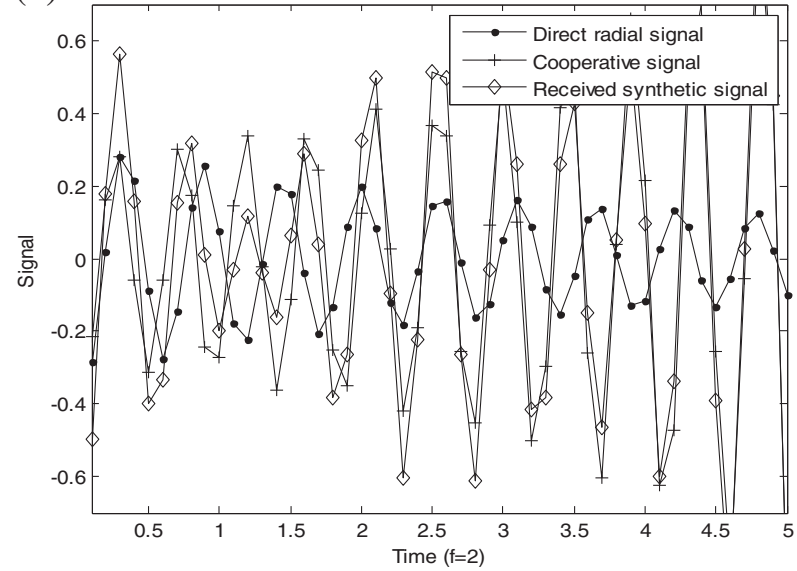

(e)

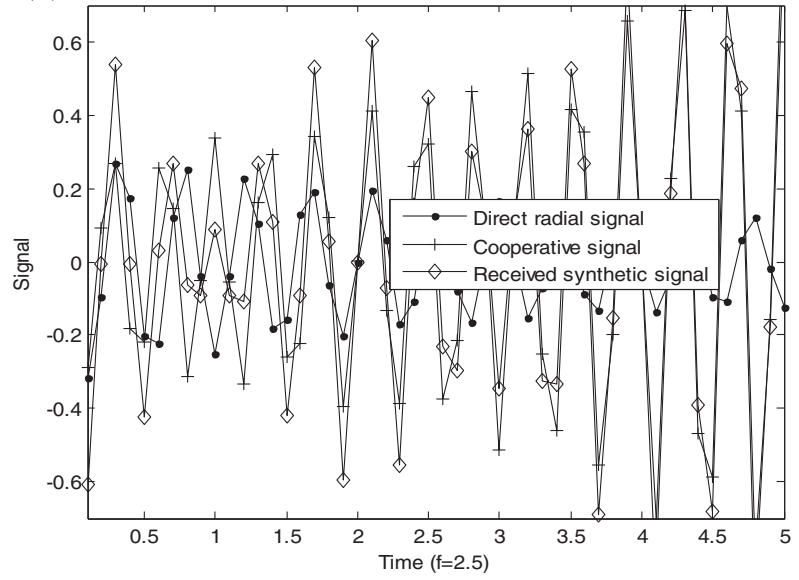

(f)

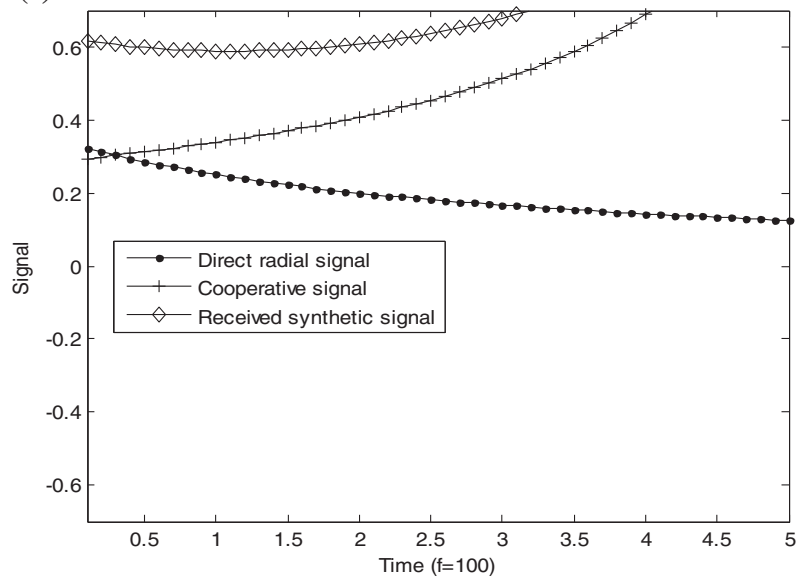

Fig. 6 a $f=0.5 \mathrm{~Hz}$. b $f=1 \mathrm{~Hz}$. $\mathbf{c} f=1.5 \mathrm{~Hz}$. $\mathbf{d} f=2 \mathrm{~Hz}$. e $f=2.5 \mathrm{~Hz}$. $\mathbf{f} f=100 \mathrm{~Hz}$

$P_{\mathrm{b}}$ is the bit error rate. When the opportunistic cooperative nodes are independent and its distribution is Gaussian, the receiver signal-to-noise ratio (SNR) could be calculated by Eq. (8).
$\left\{\begin{array}{c}\mathrm{SNR}=g\left(F_{i}\right) \\ f(x)=\frac{E_{N}}{d} \sum_{i=1}^{N_{\mathrm{M}}}\left[\mathrm{FD} * \mathrm{TD} * P_{\mathrm{b}}\right] x\end{array}\right.$ 

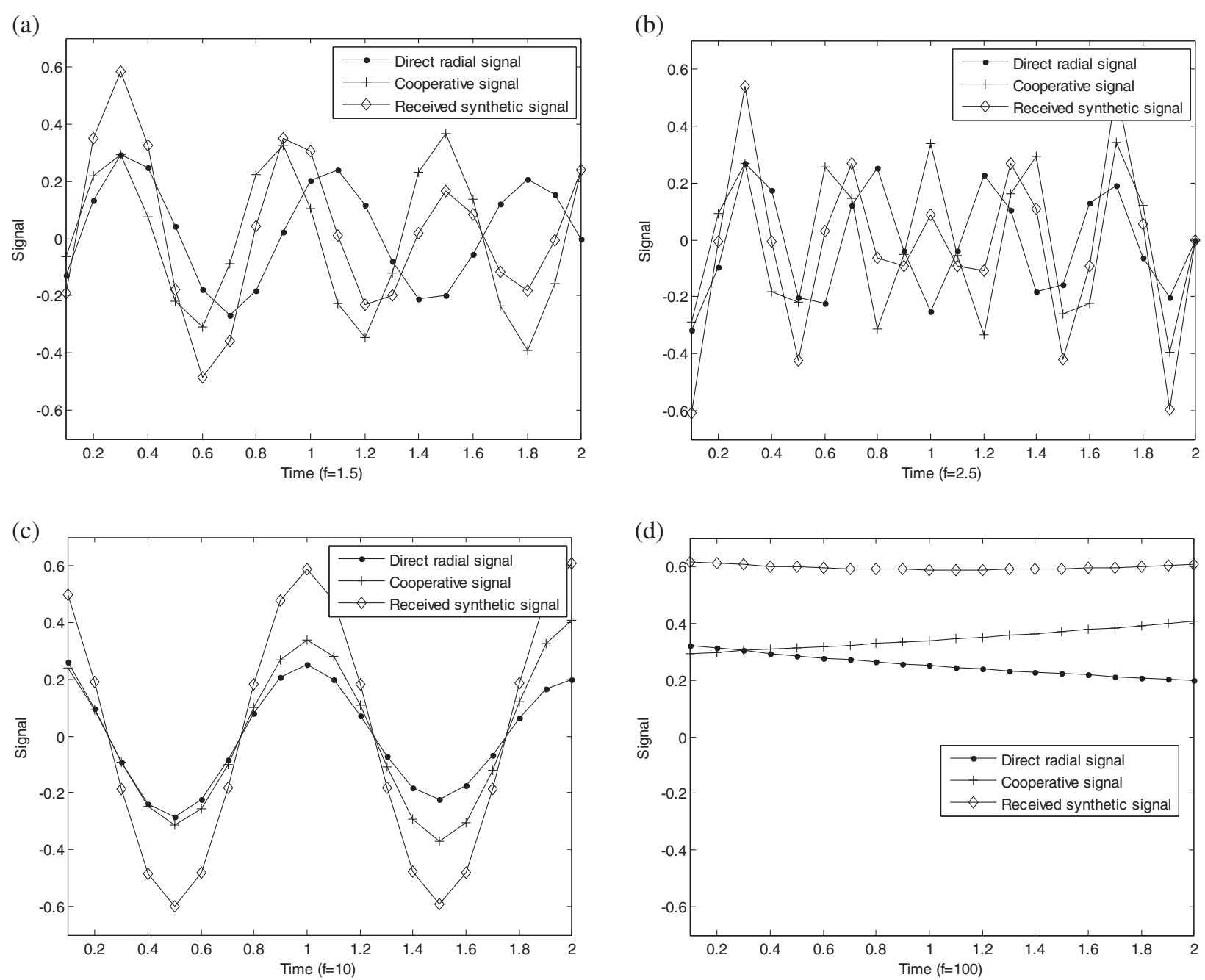

Fig. 7 a $f=1.5 \mathrm{~Hz}$. b $f=2.5 \mathrm{~Hz}$. $\mathbf{c} f=10 \mathrm{~Hz}$. $\mathbf{d} f=100 \mathrm{~Hz}$

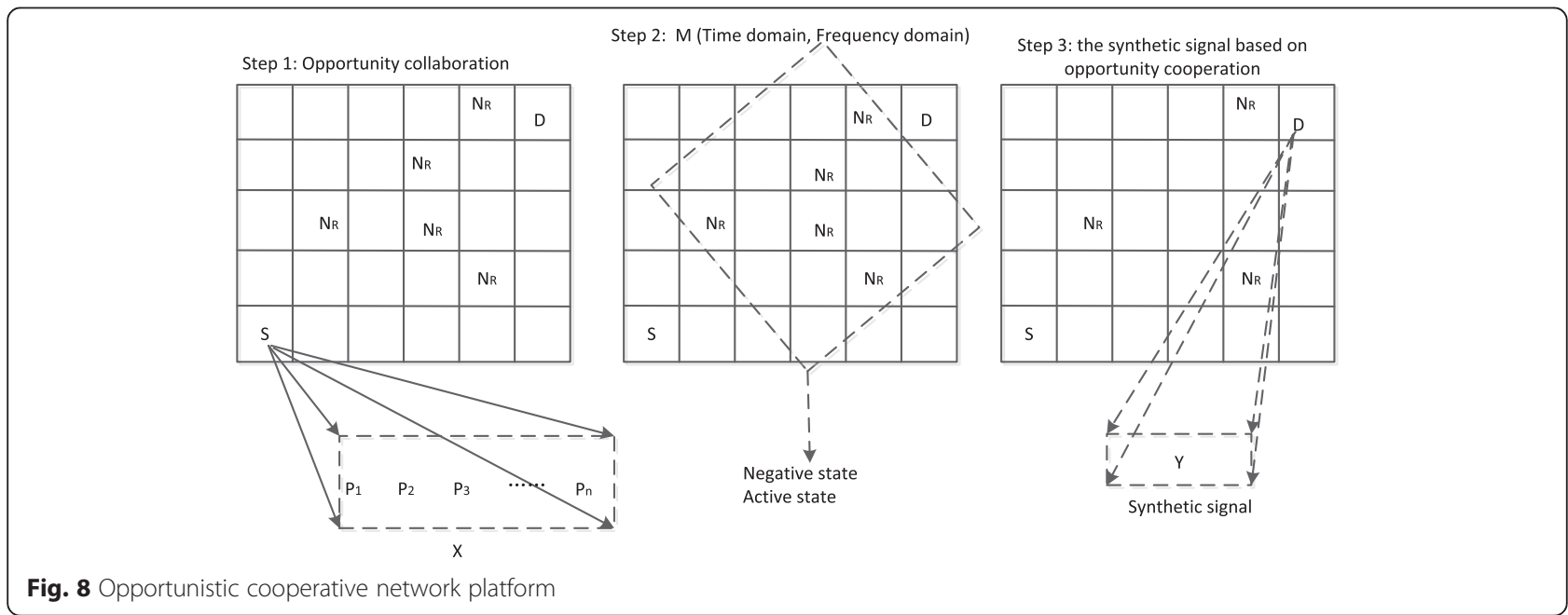

Fig. 8 Opportunistic cooperative network platform 


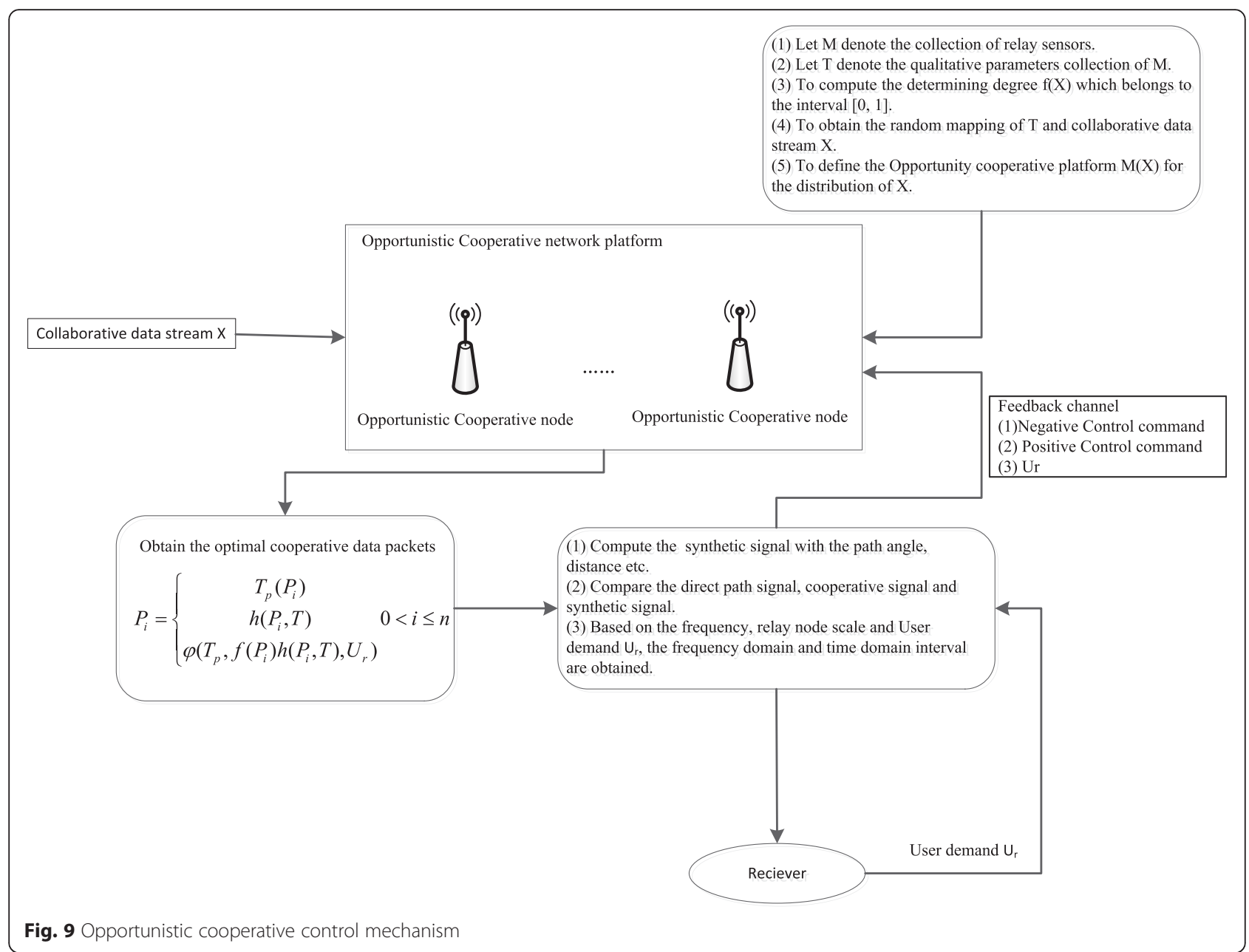

Here, $E_{\mathrm{N}}$ is the Gaussian expectations. Let $d$ denote the distance between the sender and the receiver.

The packet error rate could be calculated by Eq. (9).

$$
\left\{\begin{array}{c}
P_{M}=r\left(n, N_{\mathrm{M}}\right) \\
r(x, y)=1-\left(1-\frac{1}{2} e^{f\left(F_{i}\right)}\right) x / y \frac{\mathrm{FD} * \mathrm{TD}}{x}
\end{array}\right.
$$

The value of energy efficiency could be obtained by Eq. (10). Here, assume that $E_{\mathrm{S}}$ and $E_{\mathrm{R}}$ are the energy of sending, receiving, or transferring the cooperative data stream. Let $E_{\text {system }}$ denote the system energy consumption.

The throughput with wireless sensor networks could be obtained by Eq. (11), where $N_{\mathrm{R}}$ is the scale of cooperative sensors. Let $\phi\left(F_{m}\right)$ denote that cooperation data stream.

$$
\begin{aligned}
E_{r}(x) & =10 \ln \\
\times & {\left[\frac{L_{x}}{n E_{\text {system }}} \sqrt{\sum_{i=1}^{N_{S(x)=1}} \sum_{j=1}^{N_{S(x)=2}} \sum_{k=1}^{N_{S(x)=3}} T_{p}(x)\left[E_{R}\left(\mathrm{FD}, \mathrm{TD}, P_{b}\right)+E_{S}\left(\mathrm{FD}, \mathrm{TD}, P_{b}\right)\right]}\right] }
\end{aligned}
$$

$$
T(x)=\frac{\sum_{i=1}^{N_{S(x)=1}} \sum_{j=1}^{N_{S(x)=2}} \sum_{k=1}^{N_{S(x)=3}} T_{p}(x)\left[E_{R}\left(\mathrm{FD}, \mathrm{TD}, P_{b}\right)+E_{S}\left(\mathrm{FD}, \mathrm{TD}, P_{b}\right)\right]}{\sum_{l=1}^{L_{x}} \phi\left[(x) P_{l}\right]}
$$

We can calculate the average delay $T_{\text {avg }}$ according to Eq. (12).

$$
T_{\mathrm{avg}}=T_{p 2 p} N_{\mathrm{M}} \frac{\left(1-P_{b}^{L_{x}+1}\right)^{F D}}{1-P_{b}}
$$

Here, $T$ is the average transmission delay of single datagram.

\section{Performance evaluation and results}

In this section, the proposed opportunity cooperative platform and control mechanism based on selectivity of time domain and frequency domain (OCC-TFD), opportunity cooperative control mechanism based on selectivity of time domain (OCC-TD), opportunity cooperative control mechanism based on selectivity of frequency 
domain (OCC-FD), and cooperative control scheme based on the optimal power allocation and signal to noise (CC-OPAS) for wireless sensor networks are simulated, analyzed, and evaluated with two group experiments, which include the following metrics: signal, packet error rate, average delivery delay, throughput, and energy efficiency.

In experiment 1, the analytical results of the proposed OCC-TFD, OCC-TD, and OCC-FD schemes are illustrated as Fig. 10. From Fig. 10a, the synthetic signal of the proposed OCC-TFD is superior to the one of OCCTD and OCC-FD. The signal of OCC-TFD is two times one of OCC-TD in the best condition and is 1.2 times in the worst case. In addition, we found that the synthetic signal of OCC-TD is better than OCC-FD. That is because OCC-TD could decide the cooperative power of the relay nodes. However, the proposed OCC-TFD can consider the linear feature of the time and frequency domains for optimizing the power allocation, which could improve cooperative network efficiency and enhance the signal. As shown in Fig. 10b, the improvement of packet loss rate of OCC-FD is faster than OCC-FD. This is because the frequency domain selection scheme could adjust the transmitting frequency based on the channel quality in real time and promote the initiative of the opportunistic cooperative nodes, for ensuring the reliability of cooperative data stream. Specially, the proposed OCC-TFD has better reliability performance. When the SNR is larger than $2 \mathrm{~dB}$, the packet error rate is close to 0 . When the SNR is larger than $3 \mathrm{~dB}$, the reliability of the network reaches the best state. As shown in Fig. 10c, the proposed OCC-TFD has the optimal throughput. This benefit from the definition of cooperative data stream and opportunistic cooperative platform, which could obtain the optimal candidate data packet based on the data packet determination, data length, and user requirements. And according to the cooperative data packet redundancy degree, the priority could be computed. Based on the time domain and frequency domain, the participation enthusiasm of the opportunistic cooperation nodes in cooperative transmission would be determined, thus for keeping the high throughput and providing the reliable cooperation data stream service.

In the experiment 2 , the performance of the proposed OCC-TFD and CC-OPAS with multiple relay network topology were given by Fig. 11. Study result of delay was given by Fig. 11a. We found that the above schemes could decrease the delay with increasing the signal-tonoise ratio (SNR). However, the delay of CC-OPAS increased when the SNR is larger than $1 \mathrm{~dB}$. That is because the optimal power allocation scheme of the source node and the relay node changed. More power is allocated to the source node. So, the relay nodes have less

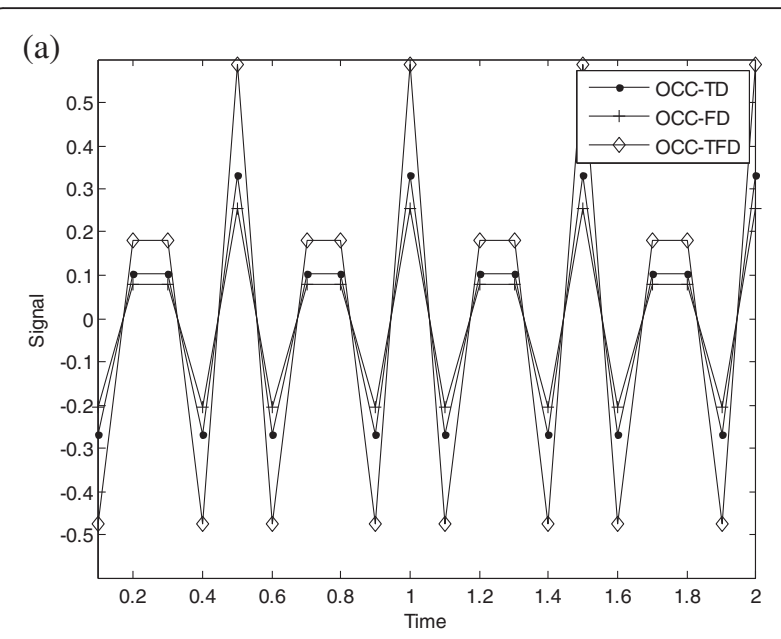

(b)

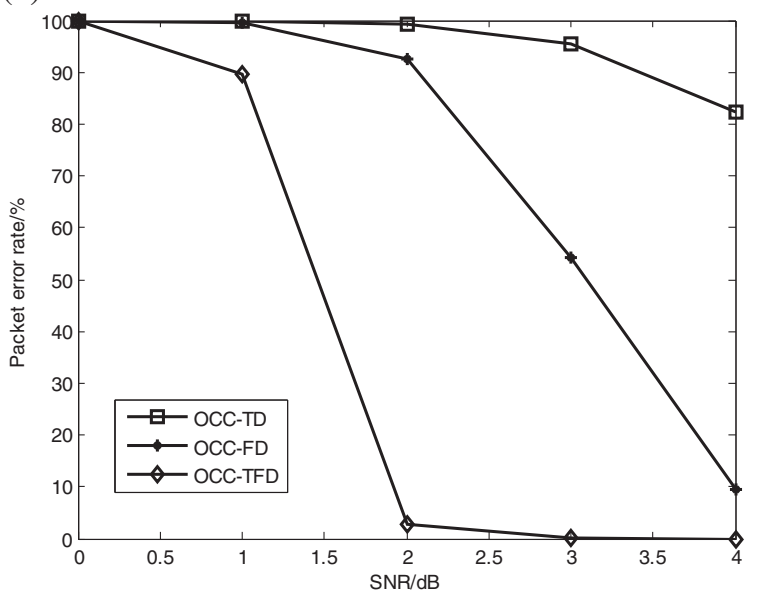

(c)

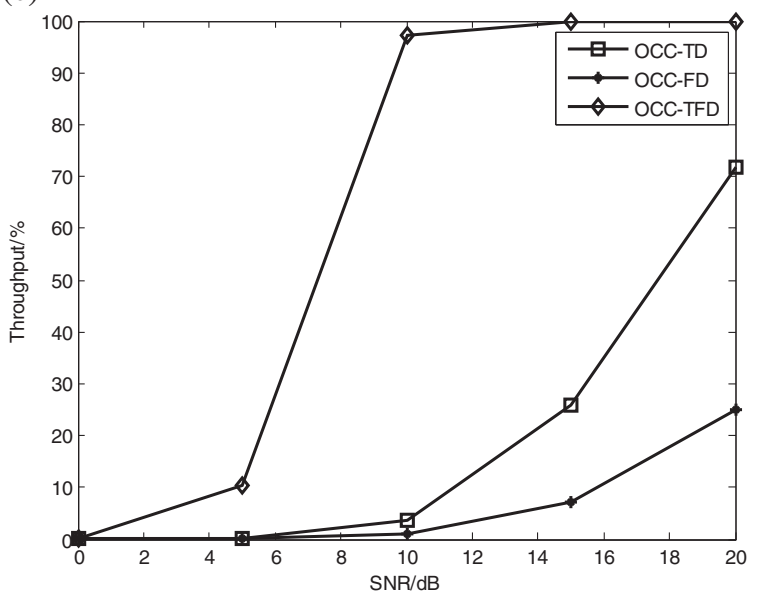

Fig. 10 a Signal. b Packet error rate. c Throughput

power, which increased the cooperative transmission delay when the SNR increased, as well as the better the quality of the channel. The proposed OCC-TFD could maintain the reducing trend of the delay and smooth the 


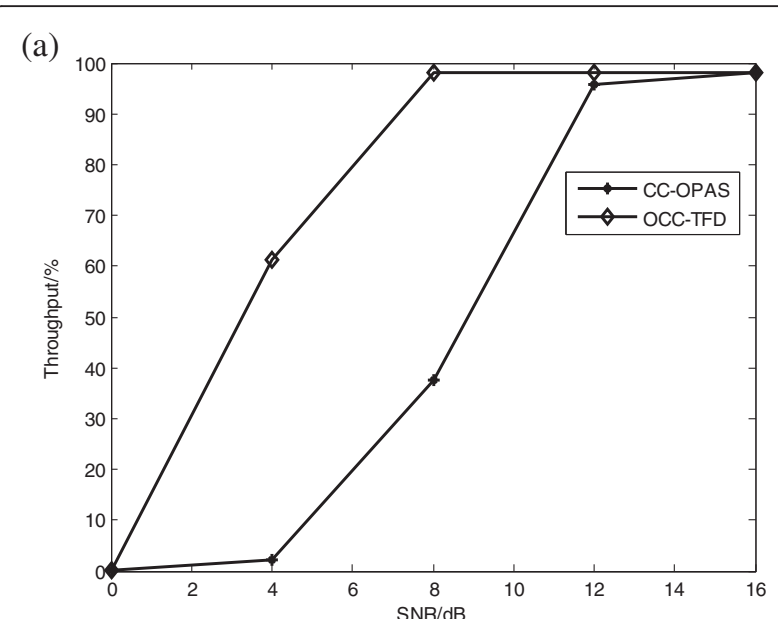

(b)

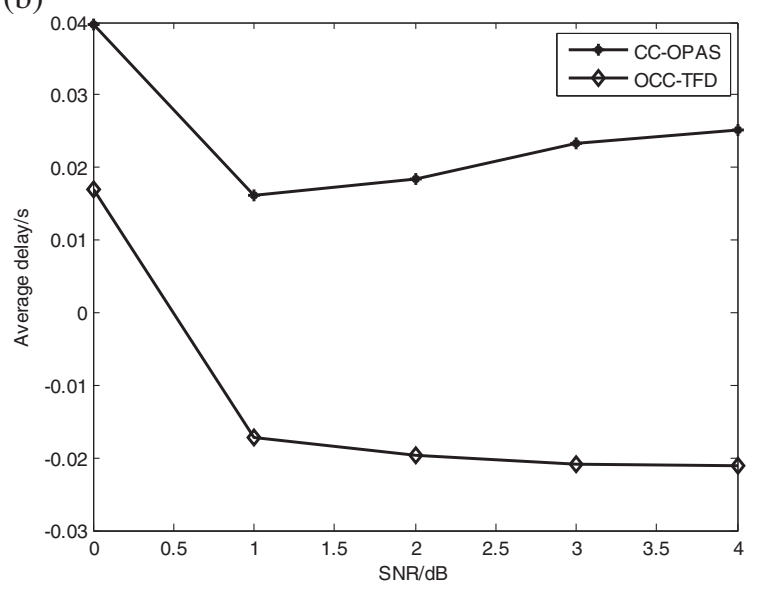

(c)

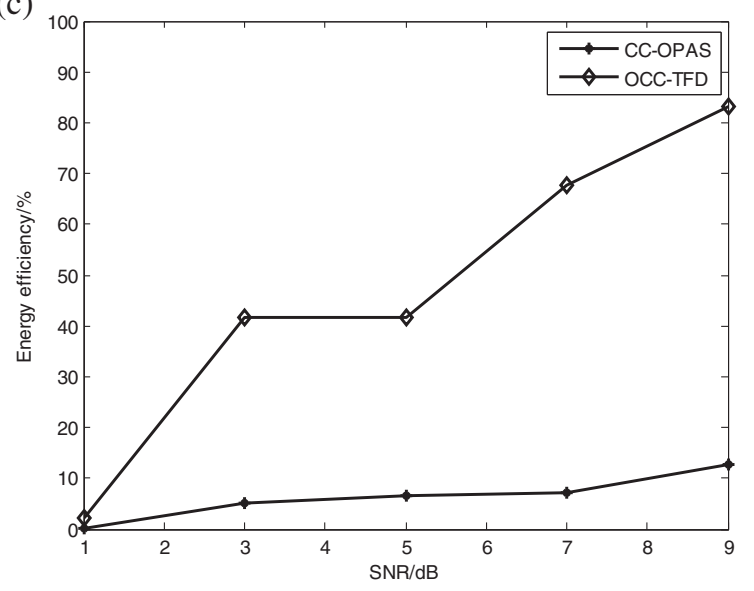

Fig. 11 a Throughput. b Delay. c Energy efficiency

delay jitter by a combination of the users' requirements, determined degree of cooperative packet, and qualitative analysis, which could map the cooperative data stream accurately and shorten the time delay.

From Fig. 11b, throughput of the proposed OCC-TFD presented the positive upward trend and reached the maximum value when $\mathrm{SNR}$ is large to $8 \mathrm{~dB}$. Although the CC-OPAS could increase the throughput, the trend is negative. Throughput of CC-OPAS reached the maximum value just only when SNR is large to $16 \mathrm{~dB}$, which is 2 times the proposed OCC-TFD. That because the time complexity and space complexity of the optimization process of power allocation result in the decrease of the system efficiency, which can affect the increase of the throughput.

As shown in Fig. 11c, energy efficiency of the proposed OCC-TFD is four times one of the CC-OPAS, which showed a rising trend with the improvement of the SNR. When the SNR is $9 \mathrm{~dB}$, the energy efficiency of OCC-TFD reached $80 \%$, but that of CCOPAS is just $10 \%$ which cannot provide reliable guarantee of long time data transmission. The proposed OCC-TFD can establish the optimal cooperative transmission collection according to the time and frequency selective characteristics of transmitter and relay nodes, to improve resource utilization and reduce energy consumption.

\section{Conclusions}

According to the cooperative data packet redundancy degree, the priority, and selective characteristics of time domain and frequency domain, we proposed the opportunistic cooperative platform and adaptive cooperative control scheme for wireless sensor networks. Based on the cooperative data stream scale, mapping scheme of data stream to users, and priority and redundancy of cooperative data packets, we defined the opportunistic cooperative platform. Then, we researched the selective characteristics of the time and frequency domains and presented the active domain and negative domain, with the single relay, multiple relay, and static and mobile network environment, which was used to adjust and improve the defined opportunistic cooperative platform. Therefore, we proposed the OCC-TFD to provide service guarantee of users in wireless sensor networks. The mathematical results indicated that the proposed platform and scheme are superior to the opportunity cooperative control mechanism based on selectivity of time domain alone and frequency domain alone, as well as cooperative control scheme based on the optimal power allocation and signal to noise, in terms of packet error rate, delay, and throughput, as well as energy efficiency.

\section{Competing interests}

The authors declare that they have no competing interests.

\section{Authors' contributions}

YJ carried out the studies of Opportunity Cooperative Embedded Platform and Control Mechanism, participated in the performance evaluation and drafted the manuscript. HD carried out the analysis of Time Domain Selectivity. CS and AL participated in the performance evaluation. PX 
participated in the analysis of Frequency Domain Selectivity. All authors read and approved the final manuscript.

\section{Authors' information}

Yong Jin was born in Shanxi Province, China, in 1981. He received the M.S. degree from the Nanjing University of Technology of China (NJUT), Nanjing, in 2009, in Computer Application Technology. He is currently working as lecturer at the Changshu Institute of Technology. His research interests include wireless sensor networks, error control, and performance evaluation. Huan Dai received a Ph.D. (2012) from the Jiang Nan University. Since 2012, he has been working at the Changshu Institute of Technology, as a lecturer at the School of Computer Science and Engineering. His research interests are in wireless communication, sensor networks, etc.

Canghai Sui and Anqi Liu are undergraduate students of the Changshu Institute of Technology. Their research interests include wireless sensor networks and Internet of Things.

Ping Xu received a Master of Engineering degree (2009) in Computer Science and Technology at the Nanjing University of Technology. Since 2009, he has been working at the Operation Research Center, Nanjing Army Command College. Her research interests are computer networks, computer algorithm, etc.

\section{Acknowledgements}

This work is supported in part by the Science and Technology Development Project of Changshu (Social Development category) Grant No. CS201413, National Natural Science Foundation of China Grant No. 61300186, and Project of Jiangsu Province University Natural Science Grant No. 13KJB510001.

\section{Author details}

${ }^{1}$ School of Computer Science and Engineering, Changshu Institute of Technology, Changshu 215500, China. ${ }^{2}$ Operation Research Center, Nanjing Army Command College, Nanjing 210045, China.

Received: 4 January 2016 Accepted: 6 March 2016

Published online: 15 March 2016

\section{References}

1. Q DAl, L RONG, H HU et al., Resource allocation using time division multiple access over wireless relay networks. J China Univ Posts Telecommun. 15, 69-74 (2008)

2. A Nayebia, H Sarbazi-Azadb, G Karlssond, Performance analysis of opportunistic broadcast for delay-tolerant wireless sensor networks. J. Syst. Softw. 83, 1310-1317 (2010)

3. N Lynn, O Takyu, K Adachi et al., An autonomous selective cooperative ARQ protocol for hybrid mobile wireless sensor networks. J Commun. 6, 157-167 (2011)

4. H Shen, G Bai, Z Tang et al., QMOR: QoS-aware multi-sink opportunistic routing for wireless multimedia sensor networks. Wireless Pers Commun (Springer) 75, 1307-1330 (2014)

5. W Prawatmuang, DKC So, E Alsusa, Sequential cooperative spectrum sensing technique in time varying channel. IEEE Trans. Wirel. Commun. 13 3393-3405 (2014)

6. FSM Vinícius, DC Felipe, FM Daniel et al., Protocols, mobility models and tools in opportunistic networks: a survey. Comput. Commun. 48, 5-19 (2014)

7. R Yuan, T Zhang, J Huang et al., Opportunistic cooperation and optimal power allocation for wireless sensor networks. IEEE Trans. Consum. Electron. 56, 1898-1904 (2010)

8. Y Shi, B Bai, J Wang, Multi-user cooperative communications scheme design based on opportunistic network coding. J Xidian Univ 38, 36-41 (2011). +53

9. M Abouelseoud, A Nosratinia, Opportunistic wireless relay networks: diversity-multiplexing tradeoff. IEEE Trans. Inf. Theory 57, 6514-6538 (2011)

10. S Zhang, $V$ Lau, Multi-relay selection design and analysis for multi-stream cooperative communications. IEEE Trans. Wirel. Commun. 10, 1082-1089 (2011)

11. Z Wang, Y Chen, C Li, CORMAN: a novel cooperative opportunistic routing scheme in mobile ad hoc networks. IEEE J Sel Area Commun. 30, 289-296 (2012)

12. X Wu, KN Brown, CJ Sreenan, Analysis of smartphone user mobility traces for opportunistic data collection in wireless sensor networks. Pervasive Mob Comput 9, 881-891 (2013)
13. H Soya, Ö Özdemir, M Bayrakc et al., Decentralized multiuser diversity with opportunistic packet transmission in MIMO wireless sensor networks. AEU Int J Electron Commun 67, 910-925 (2013)

14. Q Liang, X Cheng, SC Huang et al., Opportunistic sensing in wireless sensor networks: theory and application. IEEE Trans. Comput. 63, 2002-2010 (2014)

15. LT Lilien, L Othmane, P Angin et al., A simulation study of ad hoc networking of UAVs with opportunistic resource utilization networks. J. Netw. Comput. Appl. 38, 3-15 (2014)

16. M Orlinski, N Filer, Neighbour discovery in opportunistic networks. Ad Hoc Netw. 25, 383-392 (2015)

17. G Marzieh, A Mostafa, B Mozafar et al., Adaptive multi-flow opportunistic routing using learning automata. Ad Hoc Netw. 25, 472-479 (2015)

\section{Submit your manuscript to a SpringerOpen ${ }^{\mathcal{O}}$ journal and benefit from:}

- Convenient online submission

- Rigorous peer review

- Immediate publication on acceptance

- Open access: articles freely available online

- High visibility within the field

- Retaining the copyright to your article

Submit your next manuscript at $>$ springeropen.com 